\title{
Constraints on the Collisional Nature of the Dark Matter from Gravitational Lensing in the Cluster A2218
}

\section{Citation}

Natarajan, Priyamvada, Abraham Loeb, Jean-Paul Kneib, and lan Smail. 2002. "Constraints on the Collisional Nature of the Dark Matter from Gravitational Lensing in the Cluster A2218." The Astrophysical Journal 580 (1): L17-20. https://doi.org/10.1086/345547.

\section{Permanent link}

http://nrs.harvard.edu/urn-3:HUL.InstRepos:41393310

\section{Terms of Use}

This article was downloaded from Harvard University's DASH repository, and is made available under the terms and conditions applicable to Other Posted Material, as set forth at http:// nrs.harvard.edu/urn-3:HUL.InstRepos:dash.current.terms-of-use\#LAA

\section{Share Your Story}

The Harvard community has made this article openly available. Please share how this access benefits you. Submit a story.

Accessibility 


\title{
CONSTRAINTS ON THE COLLISIONAL NATURE OF THE DARK MATTER FROM GRAVITATIONAL LENSING IN THE CLUSTER A2218
}

\author{
Priyamvada Natarajan, ${ }^{1,2}$ Abraham Loeb, ${ }^{3}$ Jean-Paul Kneib, ${ }^{4}$ and Ian Smail ${ }^{5}$ \\ Received 2002 July 1; accepted 2002 October 15; published 2002 October 24
}

\begin{abstract}
The detailed distribution of mass within clusters of galaxies can be used to probe the nature of dark matter. We show that constraints on the extent of the mass distribution around galaxies in the rich cluster A2218 obtained from combining strong and weak lensing observations are consistent with the predictions that assume that the dominant mass component (dark matter) in these halos is collisionless. A strongly interacting (fluid-like) dark matter is ruled out at a confidence level of more than $5 \sigma$.
\end{abstract}

Subject headings: dark matter — galaxies: halos — gravitational lensing

\section{INTRODUCTION}

The detection of gravitational lensing of distant galaxies by foreground galaxies has been used in recent years to set constraints on the masses and sizes of the lensing galaxy halos. Current studies suggest the presence of halos extending beyond 100 kpc (Brainerd, Blandford, \& Smail 1996; Dell' Antonio \& Tyson 1996; Fischer et al. 2000; McKay et al. 2001; Wilson et al. 2001). The same technique can be applied to galaxies in the dense environments of massive X-ray clusters. Analysis of galaxy-galaxy lensing in the cores of rich clusters implies a smaller spatial extent for the dark matter halos associated with morphologically classified, early-type cluster members than that seen in the field (Natarajan et al. 1998). Most recently, Natarajan, Kneib, \& Smail (2002, hereafter NKS02) have found compelling evidence for the tidal truncation of dark matter subhalos in clusters based on Hubble Space Telescope (HST) imaging of lensing clusters at intermediate redshifts.

The detailed mass distribution within clusters - and in particular the fraction of the total mass that is associated with individual galaxies-has important implications for the frequency and nature of galaxy interactions. As we show in this Letter, the characteristic halo sizes of cluster galaxies that survive tidal deformation and stripping in dense environments offer tantalizing clues as to the nature of dark matter itself. While there is compelling evidence for the existence of significant amounts of dark matter in the universe, its nature remains one of the challenging and unsolved problems in cosmology. We show here that constraints on the properties of the dark matter, whether it is collisionless or collisional (fluid-like), can be derived based on the truncation radii of galaxy halos in clusters.

If the dark matter is collisionless, then the sizes of galaxy halos in clusters are primarily shaped by the dynamical effects of tidal truncation and collisional stripping (Ghigna et al. 1998). The dominant process of tidal truncation acts on the short orbital timescale due to the tidal field of the cluster as a whole (Taylor \& Babul 2001), while collisional stripping results from

\footnotetext{
${ }^{1}$ Department of Astronomy, Yale University, 260 Whitney Avenue, New Haven, CT 06511.

${ }^{2}$ Institute of Astronomy, Madingley Road, Cambridge CB3 OHA, UK.

${ }^{3}$ Department of Astronomy, Harvard University, 60 Garden Street, Cambridge, MA 02138.

${ }^{4}$ Observatoire Midi-Pyrenees, 14 Avenue Edouard Belin, 31400 Toulouse, France.

${ }^{5}$ Department of Physics, University of Durham, South Road, Durham DH1 3LE, UK.
}

binary interactions among individual galaxies (Binney \& Tremaine 1987). The global tidal field of the cluster truncates the dark matter halo of each galaxy at a radius inside of which the mean mass density of the galaxy is roughly equal to the mean interior density of the cluster. However, if the dark matter is fluid-like, then galaxy halos would be further stripped by ram pressure down to a radius that is significantly smaller than the tidal radius (roughly by the ratio between the velocity dispersion inside the galaxy halo and the velocity of the galaxy through the cluster; see Furlanetto \& Loeb 2002 for further details). In this work, we use the truncation radii of a sample of cluster galaxies, inferred observationally from the galaxygalaxy lensing in the cluster A2218 (NKS02), to decide whether the dark matter is collisionless or fluid-like.

\section{USING GALAXY-GALAXY LENSING TO MEASURE TRUNCATION RADII OF CLUSTER GALAXIES}

We model the background cluster as a superposition of a smooth large-scale potential $\left(\sim 250^{\prime \prime}\right.$ in extent) and small-scale clumps that are associated with bright early-type cluster galaxies (see Natarajan \& Kneib 1997 and Natarajan et al. 1998 for more details). To quantify the lensing distortion induced by the global potential, both the smooth component and individual galaxy-scale halos are modeled self-similarly using a surface density profile, $\Sigma(R)$, which is a linear superposition of two pseudoisothermal elliptical components (Kassiola \& Kovner 1993),

$$
\Sigma(R)=\frac{\Sigma_{0} r_{0}}{1-r_{0} / r_{t}}\left(\frac{1}{\sqrt{r_{0}^{2}+R^{2}}}-\frac{1}{\sqrt{r_{t}^{2}+R^{2}}}\right),
$$

with a core radius $r_{0}$ and a truncation radius $r_{t} \gg r_{0}$. The free parameters of this profile are chosen for both the smooth component and the clumps so as to obtain the appropriate mass distributions on the relevant scales. The projected radius $R$ is a function of the sky coordinates $x$ and $y$ and the ellipticity $\epsilon$ (see $\S 2.2$ of Natarajan \& Kneib 1997). With the additional assumption that light traces mass, galaxy halos in clusters are characterized by the following scaling laws: $\sigma=\sigma_{*}\left(L / L_{*}\right)^{1 / 4}$, $r_{0}=r_{0}^{*}\left(L / L_{*}\right)^{1 / 2}$, and $r_{t}=r_{t}^{*}\left(L / L_{*}\right)^{\alpha}$. The total mass $M_{\text {tot }}(\infty)$ then scales with the luminosity as $M_{\mathrm{tot}}(\infty)=2 \pi \Sigma_{0} r_{0} r_{t}=$ $\left(9 \sigma^{2} / 2 G\right) r_{t}=(9 / 2 G) \sigma_{*}^{2} r_{t}^{*}\left(L / L_{*}\right)^{\alpha+0.5}$, and the mass-to-light ratio $\Upsilon$ is given by $\Upsilon \sim\left(\sigma_{*} / 240 \mathrm{~km} \mathrm{~s}^{-1}\right)^{2}\left(r_{t}^{*} / 30 \mathrm{kpc}\right)\left(L / L_{*}\right)^{\alpha-0.5}$. The best-fit mass model implies $\alpha=0.5$, where $\Upsilon$ is constant for galaxies within a given $r_{t}^{*}$ and $\sigma_{*}$ (although $\Upsilon$ may be a 
TABLE 1

A2218 Clump Parameters

\begin{tabular}{|c|c|c|c|c|c|c|c|c|}
\hline Cluster & $z$ & $\begin{array}{c}x \\
(\operatorname{arcsec})\end{array}$ & $\begin{array}{c}y \\
\text { (arsec) }\end{array}$ & $\epsilon$ & $\begin{array}{c}\text { Position Angle } \\
\text { (deg) }\end{array}$ & $\begin{array}{c}\sigma \\
\left(\mathrm{km} \mathrm{s}^{-1}\right)\end{array}$ & $\begin{array}{c}r_{0} \\
(\mathrm{kpc})\end{array}$ & $\begin{array}{c}r_{t} \\
(\mathrm{kpc})\end{array}$ \\
\hline Main clump ........ & 0.17 & 2.0 & 0.3 & $0.3 \pm 0.05$ & $-13 \pm 5$ & $1070 \pm 70$ & $75 \pm 10$ & $1100 \pm 100$ \\
\hline
\end{tabular}

function of radius inside each galaxy). The parameters that characterize both the global smooth component and the clumps are optimized using the observational data (i.e., the positions, magnitudes, geometry of strong lensing features, and the smoothed shear field) as constraints.

Our goal is to optimally partition the total cluster mass between a smooth component and the clumps based on the observational data. A maximum likelihood method is used to obtain significance bounds on fiducial parameters that characterize a typical $L_{*}$ halo in the cluster. The likelihood function of the estimated probability distribution of the source ellipticities is maximized for a set of model parameters, given a functional form of the intrinsic ellipticity distribution measured for the faint background galaxies. For each faint galaxy $j$ with measured shape $\tau_{\text {obs }}$, the intrinsic shape $\tau_{S_{j}}$ can be estimated in the weak regime by subtracting the lensing distortion induced by the smooth cluster and galaxy halos,

$$
\tau_{S_{j}}=\tau_{\mathrm{obs}_{\mathrm{j}}}-\sum_{i}^{N_{\mathrm{gal}}} \gamma_{p_{i}}-\gamma_{c}
$$

where $\sum_{i=1}^{N_{c}} \gamma_{p_{i}}$ is the sum of the shear contribution at a given position $j$ from $N_{\text {gal }}$ galaxies. This entire inversion procedure is performed within the LENSTOOL utilities developed by Kneib (1993), which accurately take into account the nonlinearities arising in the strong lensing regime. Using a well-constrained "strong lensing" model for the inner regions of the cluster along

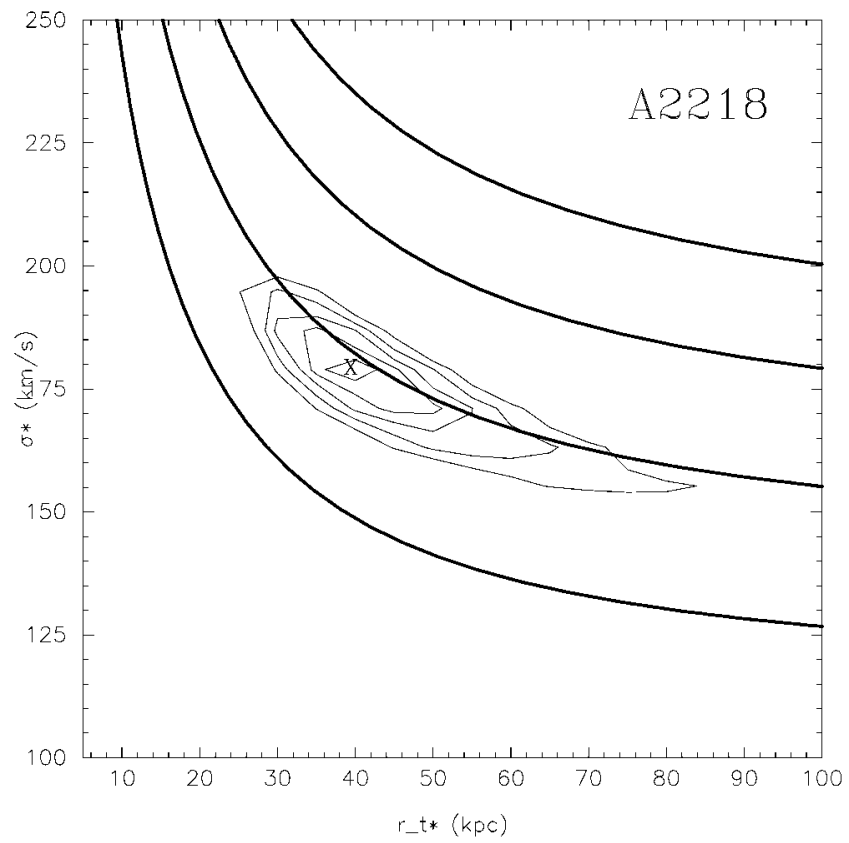

FIG. 1.-Results of maximum likelihood analysis of lensing data for the cluster A2218 (NKS02). The contour plot shows the best-fit values for the model parameters $\sigma_{*}$ and $r_{t}^{*}$, which are the central velocity dispersion and truncation radius for a typical $L_{*}$ galaxy in the cluster. The likelihood contours are plotted in intervals of $1 \sigma$ starting from the inside out. The thick curves are lines of constant enclosed mass. with the averaged shear field and assuming a known functional form for $p\left(\tau_{S}\right)$ from the field, the likelihood for a guessed model is

$$
\mathcal{L}\left(\sigma_{*}, r_{t}^{*}\right)=\Pi_{j=1}^{N_{\text {gal }}} p\left(\tau_{S_{j}}\right) .
$$

We compute $L$ by assigning the median redshift corresponding to the observed source magnitude for each arclet. The best-fitting model parameters are then obtained by maximizing the log likelihood function with respect to the parameters $\sigma_{*}$ and $r_{t}^{*}$. Using a bootstrap method, we have verified that while the convergence to the best-fit model is indeed driven by the brighter cluster galaxies, no single galaxy dominates in the procedure.

\section{PROPERTIES OF A2218}

The lens model for the mass distribution in the well-studied cluster A2218 at $z=0.17$ is arguably the best-constrained case presently available (Kneib et al. 1996) to which we can apply our analysis. We use four sets of multiple images, which are all spectroscopically confirmed at $z=0.70,1.03,2.52$, and 5.60 , in conjunction with the background shear field, to obtain the best-fit parameters for a fiducial $L_{*}$ galaxy, $r_{t}^{*}=40_{-10}^{+30} \mathrm{kpc}$ and $\sigma^{*}=180_{-20}^{+15} \mathrm{~km} \mathrm{~s}^{-1}$ (where the quoted error bars are at $5 \sigma$ confidence). The mass distribution in A2218 is composed of two clumps centered around the two brightest cluster galaxies, and the profile inferred from lensing observations and $\mathrm{X}$-ray data are consistent. Although the mass distribution is bimodal, we note that the primary clump contributes $\sim 90 \%$ of the mass. Nevertheless, we take the presence of the second clump into account when calculating the center of mass of the cluster. In Table 1, we tabulate the model parameters for these two components in our best-fit mass model of A2218.

The morphologies and luminosities of the cluster members (which are crucial for the lensing analysis) are also well determined from the high-resolution HST imaging of this cluster (Smail et al. 2001). We have used the 40 brightest early-type cluster galaxies in the maximum likelihood analysis to obtain the values quoted above (see Fig. 1). Their individual truncation radii were then determined by utilizing the scaling laws with luminosity. Out of the early-type cluster galaxies selected for the lensing analysis, $\sim 75 \%$ are located inside the core radius and the rest lie interior to $300 \mathrm{kpc}$, within a few core radii from the cluster center.

There have been several dynamical studies of A2218 that yielded accurate measurements of galaxy velocities relative to the center of mass of the cluster (Danese, de Zotti, \& di Tullio 1980; Le Borgne, Pello, \& Sanahuja 1992; Girardi et al. 1997; Rakos, Dominis, \& Steindling 2001). Using the spectrophotometric survey of Le Borgne et al. (1992), we find that 25 of the 40 early-type galaxies in our analysis have measured redshifts that allow us to estimate their velocity relative to the center of mass of the cluster. We have then computed the expected truncation radii for these galaxies in two limiting cases regarding the composition of their dark matter halos: (1) col- 


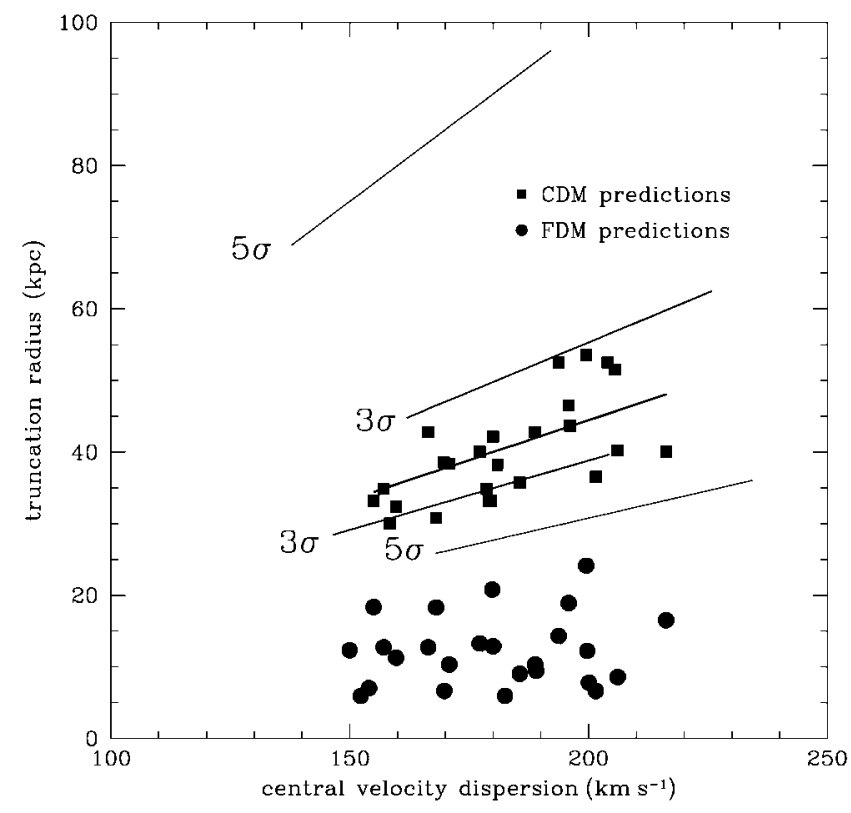

FIG. 2.-Distribution of truncation radii as inferred from the lensing analysis of 25 galaxies superposed on the scaling relation (thick solid line) in the cluster A2218. The $3 \sigma$ and $5 \sigma$ lines denote the corresponding confidence levels in the parameters $r_{t}^{*}$ and $\sigma^{*}$ obtained for a typical $L_{*}$ galaxy in this cluster (derived from the confidence level contours in Fig. 1). The expected distribution of tidal radii for FDM (filled circles) and collisionless CDM (filled squares) are also shown. Note that the linear relation between truncation radius and velocity dispersion for the values inferred from lensing is a consequence of the assumed scaling laws with galaxy luminosity (eq. [2]).

lisionless dark matter and (2) fluid-like dark matter (FDM; see Furlanetto \& Loeb 2002).

In our analysis, we have to additionally assume that the orbits of the cluster galaxies under consideration are representative of tidally stripped galaxies. Ghigna et al. (1998) have found that most of the strongly tidally stripped halos move on nearly radial orbits (since these orbits penetrate the high-density core of the cluster) and that radial orbits are overabundant by an order of magnitude relative to tangential orbits in the inner $r \$ 500 \mathrm{kpc}$ of the cluster. Since we are using the projected location of cluster galaxies in conjunction with their orbital velocities at the present time, the nature of the orbits interior to $300 \mathrm{kpc}$ needs to be established in order to justify the assumption that these galaxies are indeed populating representative orbits for efficient tidal stripping. Using the determined mass profile of the cluster, the lineof-sight velocity dispersion profile, and the number density profile of the galaxies, and assuming that cluster galaxies are good tracers of the potential well, we can compute the velocity anisotropy parameter, ${ }^{6} \beta(r)=\left(1-\sigma_{t}^{2} / \sigma_{r}^{2}\right)$, via the anisotropic Jeans equation using the approximation of spherical symmetry (for further details see Natarajan \& Kneib 1996),

$$
\frac{d\left(\nu_{g} \sigma_{r}^{2}\right)}{d r}+\frac{2 \beta(r) \nu_{g} \sigma_{r}^{2}}{r}=-\frac{G M_{\mathrm{tot}}(r) \nu_{g}}{r^{2}}
$$

where $\nu_{g}(r)$ is the three-dimensional galaxy density profile (as derived from the two-dimensional projected distribution via the Abel transform), $\sigma_{r}^{2}(r)$ is the radial velocity dispersion of the galaxies, and $M_{\mathrm{tot}}(r)$ is the total mass profile. The observed

\footnotetext{
${ }^{6}$ Note that $\beta=0$ implies isotropic orbits, $0<\beta \leq 1$ implies mostly radial orbits, and $\beta<0$ indicates orbits that are primarily tangential.
}

line-of-sight velocity dispersion profile $\sigma_{\text {los }}(R)$ is given by

$$
\frac{1}{2}\left[\Sigma_{g}(R) \sigma_{\mathrm{los}}^{2}(R)\right]=\int_{R}^{\infty} \frac{r \nu_{g}(r) \sigma_{r}^{2}(r) d r}{\sqrt{\left(r^{2}-R^{2}\right)}}\left[1-\frac{R^{2}}{r^{2}} \beta(r)\right] .
$$

The above two integrodifferential equations can be solved numerically for $\sigma_{r}^{2}$ and $\beta(r)$. Substituting the best-fit mass model for $\mathrm{A} 2218$, we obtain $\beta \approx 0.3$, which indicates that the majority of orbits are in fact radial and do plunge through the core of the cluster. Such orbits are likely to facilitate tidal truncation, as assumed in our analysis.

\section{RESULTS}

For collisionless cold dark matter (CDM), the tidal truncation radius, $r_{t}$, for the $i$ th galaxy whose pericenter lies at a radial position $r_{i}$ inside the cluster, is defined by the condition that the average density of the galaxy halo interior to the tidal radius matches the mean density of the cluster interior to the galaxy location $r_{i}$ (Ghigna et al. 1998; Taylor \& Babul 2001):

$$
\left\langle\rho_{g_{i}}\left(r_{t_{i}}\right)\right\rangle=\left\langle\rho_{\mathrm{cl}}\left(r_{i}\right)\right\rangle \text {. }
$$

For the case of FDM, the halo truncation process is modified owing to the additional process of ram pressure stripping. In this case, the truncation radius is set by the condition (Furlanetto \& Loeb 2002)

$$
\rho_{c}\left(r_{i}\right) v_{g_{i}}^{2}=\rho_{g_{i}}\left(r_{t}\right) \sigma_{g_{i}}^{2},
$$

where $v_{g_{i}}$ is the velocity component of the $i$ th galaxy relative to the cluster center of mass, $\rho_{g_{i}}\left(r_{t}\right)$ is the density of that galaxy halo at its three-dimensional truncation radius $r_{t}$, and $\sigma_{g_{i}}$ is the internal velocity dispersion of that galaxy. ${ }^{7}$ In our analysis, we have conservatively used only the measured component of the velocity of each galaxy (relative to the cluster center) along line of sight. Since the full (three-dimensional) galaxy velocity can only be larger than our adopted value, our constraint on the truncation radii of galaxies in the FDM case should be regarded as conservative upper limits. The considerations quoted above ignore any further truncation that is likely to be caused by thermal or turbulent heating (Gnedin \& Ostriker 2001). Using the above expressions, we derived the truncation radii for the 25 early-type galaxies in A2218 in the two limiting cases. The results are plotted in Figure 2. The observed distribution of truncation radii from the lensing data analysis is compared to the radii expected in the collisionless limit (filled squares) and the fluid limit (filled circles). We find that FDM models are ruled out at a confidence greater than $5 \sigma$, while collisionless dark matter models are in excellent agreement with the observationally determined values. We find no correlation between the truncation radii and the orbital velocities for the cluster members in A2218, as would be expected if ram pressure stripping had been a significant dynamical process in operation.

The association of the retrieved model radius $r_{t}^{*}$ from the lensing analysis with the tidally truncated radius is strengthened

\footnotetext{
${ }^{7}$ Note that while the truncation radii inferred from the lensing observations are projected two-dimensional values, those calculated from eqs. (7) and (8) are in three dimensions. However, because the pseudoisothermal elliptical mass distribution (PIEMD) mass density profile is $\rho \propto\left(r^{2}+r_{0}^{2}\right)^{-1}\left(r^{2}+r_{t}^{2}\right)^{-1}$, both truncation radii are equivalent.
} 
through the following observed correlation. For a galaxy described by a PIEMD mass profile with velocity dispersion $\sigma_{0_{i}}$ and whose orbital pericenter crosses the cluster core where the density is $\rho_{0}$, the tidal truncation radius scales as $r_{t} \propto \sigma_{0_{i}} \rho_{0}^{-1 / 2}$.

Deriving the best-fit values of $\rho_{0}$ from the strong lensing models and $\sigma_{0_{i}}$ from the maximum likelihood analysis, we find that the above simple scaling adequately reproduces the observed trends both for the truncation radius and the mass of a fiducial halo in the five HST clusters studied in NKSO2 (ranging in redshift from $z=0.17$ to 0.58 ). The trends seen in halo size $r_{t}^{*}$ with redshift from the lensing study of NKSO2 are also in good agreement with the theoretical expectation from numerical simulations (Ghigna et al. 1998). High-resolution collisionless $\mathrm{N}$-body simulations of cluster formation and evolution predict that the dominant interaction is between the global cluster tidal field and individual galaxies in the redshift interval $z \sim 0-0.5$ (Ghigna et al. 1998; Moore et al. 1996).

\section{CONCLUSIONS AND DISCUSSION}

We have used gravitational lensing data to constrain the truncation radii of galactic dark matter halos in the cluster A2218. Figure 2 shows that the inferred truncation radii are consistent with the tidal radii expected for collisionless dark matter, but rule out FDM for which ram pressure stripping is effective. The transition between the collisionless and collisional regimes is set by the ratio between the mean free path of dark matter particles, $\lambda$, and the radius, $r_{t}$, of the galaxy halos under consideration. This ratio is given by

$$
\frac{\lambda}{r_{t}} \approx \frac{m_{p}}{\sigma_{p} \Sigma\left(r_{t}\right)},
$$

where $\sigma_{p} / m_{p}$ is the collisional cross section per unit particle mass and $\Sigma\left(r_{t}\right)$ is the surface mass density of a galaxy halo at its truncation radius. The fluid regime is obtained for $\lambda / r_{t} \lesssim 1$. The characteristic surface mass densities of the galaxies in A2218 can be directly inferred from the analysis of the lensing data. We find that for an $L_{*}$ galaxy, $\Sigma_{*}\left(r_{t}\right) \approx 0.024 \mathrm{~g} \mathrm{~cm}^{-2}$. Since the fluid regime is ruled out, we exclude all values of $\sigma_{p} / m_{p} \gtrsim$ $42 \mathrm{~cm}^{2} \mathrm{~g}^{-1}$.

Our constraints are complementary to those derived by Gnedin \& Ostriker (2001) from considerations of thermal conduction in the mildly collisional regime. These authors exclude the regime $0.3 \lesssim \sigma_{p} / m_{p} \lesssim 10^{4} \mathrm{~cm}^{2} \mathrm{~g}^{-1}$, based on the consideration that cluster elliptical galaxies will otherwise deviate from the fundamental plane beyond the observed scatter. Our new constraint allows us to rule out the high cross section regime, and hence we conclude that $\sigma_{p} / m_{p} \lesssim 0.3 \mathrm{~cm}^{2} \mathrm{~g}^{-1}$ is an absolute upper limit. Dark matter cross sections higher than this upper limit were postulated by Spergel \& Steinhardt (2000) in order to reconcile problems that CDM models possess when compared to observational data (such as the abundance of galactic subhalos and the slope of the inner mass density profile of galaxies; see further discussion in Davé et al. 2001; Yoshida et al. 2000; Stoehr et al. 2002; Miralda-Escudé 2002).

P. N. acknowledges support from a Trinity College Research Fellowship. This work was supported in part by NSF grants AST-9900877 and AST-0071019 for A. L.; J.-P. K. thanks the CNRS and the TMR-Lensing collaboration and I. S. the Royal Society and the Leverhulme Trust for support.

\section{REFERENCES}

Binney, J., \& Tremaine, S. 1987, Galactic Dynamics (Princeton: Princeton Univ. Press), chap. 7

Brainerd, T., Blandford, R., \& Smail, I. 1996, ApJ, 466, 623

Danese, L., de Zotti, G., \& di Tullio, G. 1980, A\&A, 82, 322

Davé, R., Spergel, D., Steinhardt, P. J., \& Wandelt, B. 2001, ApJ, 547, 574

Dell'Antonio, I., \& Tyson, J. A. 1996, ApJ, 473, L17

Fischer, P., et al. 2000, AJ, 120, 1198

Furlanetto, S., \& Loeb, A. 2002, ApJ, 565, 854

Ghigna, S., Moore, B., Governato, F., Lake, G., Quinn, T., \& Stadel, J. 1998, MNRAS, 300, 146

Girardi, M., Fadda, D., Escalera, E., Giuricin, G., Mardirossian, F., \& Mezzetti, M. 1997, ApJ, 490, 56

Gnedin, O., \& Ostriker, J. P. 2001, ApJ, 561, 61

Kassiola, A., \& Kovner, I. 1993, ApJ, 417, 474

Kneib, J.-P. 1993, Ph.D. thesis, Univ. Paul Sabatier, Toulouse

Kneib, J.-P., Ellis, R. S., Couch, W., Smail, I. R., \& Sharples, R. 1996, ApJ, 471,643

Le Borgne, J.-F., Pello, R., \& Sanahuja, B. 1992, A\&AS, 95, 87
McKay, T. A., et al. 2001, ApJ, submitted (astro-ph/0108013)

Miralda-Escudé, J. 2002, ApJ, 564, 1019

Moore, B., Katz, N., Lake, G., Dressler, A., \& Oemler, A. 1996, Nature, 379, 613

Natarajan, P., \& Kneib, J.-P. 1996, MNRAS, 283, 1031 1997, MNRAS, 287, 833

Natarajan, P., Kneib, J.-P., \& Smail, I. 2002, ApJ, 580, L11 (NKS02)

Natarajan, P., Kneib, J.-P., Smail, I., \& Ellis, R. S. 1998, ApJ, 499, 600

Rakos, K., Dominis, D., \& Steindling, S. 2001, A\&A, 369, 750

Smail, I., Kuntschner, H., Kodama, T., Smith, G. P., Packham, C., Fruchter, A. S., \& Hook, R. N. 2001, MNRAS, 323, 839

Spergel, D., \& Steinhardt, P. J. 2000, Phys. Rev. Lett., 84, 3760

Stoehr, F., White, S. D. M., Tormen, G., \& Springel, V. 2002, MNRAS, 335, L84

Taylor, J. E., \& Babul, A. 2001, ApJ, 559, 716

Wilson, G., Kaiser, N., Luppino, G. A., \& Cowie, L. L. 2001, ApJ, 555, 572

Yoshida, N., Springel, V., White, S. D. M., \& Tormen, G. 2000, ApJ, 535, L103 